\section{Telephone scam hits oral health charity}

The Dental Helpline service operated by the Oral Health Foundation has been exploited by scammers who have illegally taken money from callers, it has emerged.

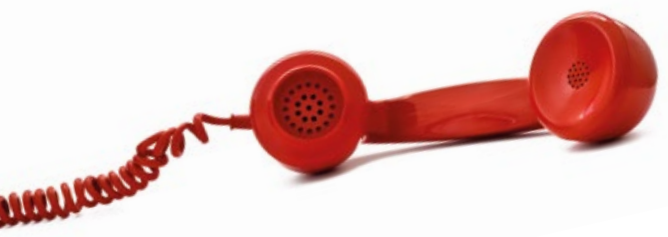

The Foundation has alerted the public and dental professionals to the fact that scammers have created bogus premium line numbers claiming to be run by the Foundation but which take money from unsuspecting callers.

These bogus numbers claim to forward people to the Dental Helpline and when connected, callers are then open to highly-inflated charges. These additional and illicit charges have been collected by criminals and not passed onto the charity.

The Oral Health Foundation said it wanted to clarify that its Dental Helpline could only be called on its local-rate Rugby telephone number - 01788539 780. Any other telephone numbers claiming to be its Dental Helpline (including those beginning with 0845) were not genuine.

Foundation Chief Executive Dr Nigel Carter OBE said: 'We are aware of a telephone scam in which a series of fake numbers, claiming to be the Dental Helpline, are taking money from unsuspecting callers.

'The welfare of those who reach out to our Dental Helpline is really important to us. It is always best to check the validity of a helpline number before calling or signposting people towards it. This is especially true if it is about a person's health. In this case, their financial safety might be at risk too. The Helpline can also be reached by email at helpline@dentalhealth.org or via the website at www.dentalhealth.org/ dentalhelpline.

\title{
Almost all child hospital tooth extractions caused by decay
}

Almost all (nine out of ten) hospital tooth extractions among children aged 0 to 5 are due to preventable tooth decay, suggests new data ${ }^{1}$ published by Public Health England (PHE).

The data published on 6 March 2019 also showed significant variation in rates of decay in children when comparing areas by affluence with children in parts of Yorkshire and the North West five times more likely to undergo hospital extractions than the national average.

PHE said oral health of children was improving but significant inequalities remained.

The data on hospital-based tooth extractions for children covering every local authority in England in 2017-18 show there were 59,314 tooth extractions in 2017-2018 (38,385 extractions because of tooth decay), slightly less than in 2016-2017 when the total number of tooth extractions was 61,301 (39,010 extractions because of tooth decay).

However, among 0 to 5 -year-olds, there were 14,545 tooth extractions in 2017-2018 $(12,783$ because of tooth decay, or $87.9 \%)$. In 2016-2017, the statistics were for 0 to 4 -yearolds, so cannot be directly compared.

Although the total actual numbers of tooth extractions fell slightly since last year, the percentage of extractions as a proportion of the population has remained the same as in previous years $-0.3 \%$ of the 0 to 19 -year-old population.

While children's oral health was improving with $77 \%$ of 5-year-old children in England now free of obvious decay, children from deprived areas had more than twice the level of decay (34\%) than those from the least deprived areas (14\%).

PHE said tooth decay could be prevented by cutting down on sugar and practising good oral hygiene and although children's sugar intakes had declined in recent years, they were still consuming the equivalent of around eight sugar cubes more than recommended daily limits.

To tackle this, PHE's Change4Life campaign was encouraging parents to 'Make a swap when you next shop' and switch to lower sugar alternatives.

Dr Sandra White, Dental Lead for PHE, said: 'Children are consuming far too much sugar each day, and this can have a very serious impact on their oral health.
'Parents can help reduce their children's sugar intake by making simple swaps when shopping and making sure their children's teeth are brushed twice a day with fluoride toothpaste.'

The BDA said the government had to tackle the deep-seated oral health inequalities across England.

The worst affected areas included Rotherham, Sheffield, Preston and Blackpool. Doncaster had England's highest rates of extractions (nearly 600 in total), more than five times the national average for 6-10-yearolds undergoing the procedure.

The BDA pointed to PHE's own modelling which showed that interventions in deprived areas could yield significant returns on investment. The figures show $£ 1$ spent on supervised tooth brushing programmes in nurseries and primary schools would yield a $£ 3.06$ return in five years, rising to $£ 3.66$ in ten, as a result of reduced treatment costs.

BDA Chair Mick Armstrong said: 'Children's oral health shouldn't be a postcode lottery, but these figures show just how wide the oral health gap between rich and poor has become.

'While Wales and Scotland have national programmes making real inroads, in England ministers are yet to commit a penny of new money to the challenge. This poverty of ambition is costing our NHS millions, even though tried-and-tested policies would pay for themselves.

'The government's own figures show a pound spent on prevention can yield over three back in savings on treatment.'

Dr Max Davie, Officer for Health Improvement at the Royal College of Paediatrics and Child Health, said: Action is needed at a national, local, and individual level to tackle the postcode lottery in children's oral health, and reduce the number of children having to endure uncomfortable tooth extractions, often under general anaesthetic.'

\section{References}

1. Public Health England. Hospital tooth extractions of 0 to 19 year olds. 2019. Available at https://www.gov.uk/ government/publications/hospital-tooth-extractions-of0 -to-19-year-olds (accessed March 2019).

2. Public Health England. Return on investment of oral health improvement programmes for 0-5 year olds. 2019. Available at https://assets.publishing.service. gov.uk/government/uploads/system/uploads/attachment_data/file/560973/ROI_oral_health_interventions. pdf (accessed March 2019). 